\title{
Utilizing 3D Laser Scanning Technology for Remodeling Work of Building Inside
}

\author{
Jin-Duk Lee \\ School of Civil and Environmental Engineering \\ Kumoh National Institute of Technology, Gumi, Gyeongbuk 730-701, Korea \\ Seung-Hee Han \\ Department of Civil Engineering \\ Kongju National University, Cheonan, Chungnam 330-717, Korea \\ Jae-Bin Lee \\ Dongsan Survey ENC, Seoul 137-131, Korea
}

\begin{abstract}
Laser scanning technology is a maturing measurement technology which is capable of obtaining $3 D$ measurement data of objects with high-accuracy, high-resolution and in a short time. Laser scanners are used more and more as surveying instruments for various applications. This paper describes the procedure of $3 D$ data acquirement using terrestrial LiDAR and section drawing extraction through a series of processing for remodeling the interior of a department building.

Accurate drawings are needed for improvement construction of building interior. However if the design drawings of that time of construction work were lost or damaged or actual dimensions of drawings differ from those of design drawings, the interior should be resurveyed. In this study, the extraction process of interior plane figures were suggested through using laser scanning and related reverse engineering software
\end{abstract}

Keywords: Laser Scanner, Reverse Engineering, 3D Measurementt and Plane Figure., Inside Improvement, Terrestrial

\section{INTRODUCTION}

The market of laser scanners for terrestrial applications has developed over the last years quite successfully and the laser scanners are seen as surveying instruments which meet the requirements of industrial applications. Traditional surveying instruments, CMM(coordinate-measuring machine) and so forth have been used for $3 \mathrm{D}$ digital data of objects. A CMM is limited in a installation position and require much time and skill for measurement, while a laser scanner is able to acquire data very simply and in a short time because it is measures automatically by a non-contact manner ${ }^{1)}$.

Laser scanning technology is a maturing measurement technology which is capable of obtaining high-accuracy and high-resolution 3D data in a short time. The data collected by laser scanners are point-clouds representing 3D coordinates of points which reflect the laser signals back to the receiver ${ }^{2}$.

The objective of 3D scanning is to restitute the accurate shapes of objects in space, store them as digital data and obtain

This is an excellent paper selected from the papers presented at ICCC 2008.

${ }^{*}$ Corresponding author. E-mail : jdlee@kumoh.ac.kr

Manuscript received Mar. 11, 2009; accepted Jun. 15, 2009 various data through the stored data. That is, 3D scanning is a series of process for restituting a $3 \mathrm{D}$ model with actual dimensions by generating surface using polygon data formed from point-cloud and acquiring various data and information.

The reverse engineering is the technique which restitute shape and is composed of steps which measure objects, transform measured data into structured point-cloud, and transform point-cloud into 3D CAD model or 3D model with texture. The reverse engineering is in great demand in the industry at large including not only manufacturing industry such as automobile, aero space or electric home appliance which need making 3D models promptly and accurately but also cultural industry such as advertisement, movie and so on which intend to obtain lifelike 3D models ${ }^{8)}$.

Field surveyors became to get a great number of point-cloud data on the surface of objects in a short time by means of a $3 \mathrm{D}$ laser scanner equipment which is also called Terrestrial LiDAR(light detection and ranging). In Korea, a terrestrial LiDAR has been utilized for volume measurement of a underground cave, deformation measurement of a tunnel, a retaining wall, etc, design of steel box girder bridge and slope stability, preservation and restoration of cultural assets and 
modeling of buildings and structures.

Also combining a terrestrial laser scanner and digital terrestrial photogrammetry, the models of old architectures were generated and through the construction project of overall information system of national cultural assets, which carried out by support of Ministry of Culture, Sports \& Tourism in Korea, 3D digital data were completed for about 200 main antiquities in the possession of 12 national museums and 25 public and private museums.

Terrestrial laser scanners have been utilized in wide area such as transportation section in Traffic Department of USA IOWA State, structural monitoring, recording and modelling of old infrastructure and cultural asset conservation state, monitoring of land slide and glaciers, observation of facility around rail track, and structural measurement of hydro power plant and so forth. In this research, we suggested plane figures extraction procedure for remodeling execution of a building interior through a series of processing of data acquired by a terrestrial laser scanner.

\section{DATA ACQUISITION}

\subsection{Specifications}

A $\mathrm{Z}+\mathrm{F}$ 3D Laser Scanner used in this research is capable of measuring the maximum of 500,000 points per second at accuracy upto at least $0.1 \mathrm{~mm}$ with rotation upto the range of $360^{\circ}$ arc horizontally and $310^{\circ}$ arc vertically, and the maximum observation distance of 79 meter as you see in Table $1^{7)}$.

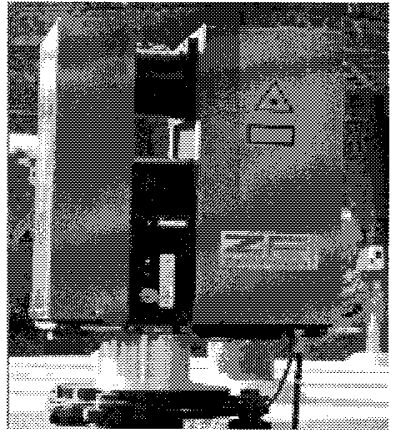

Fig. 1. Z+F 3D laser scanner

Table 1. Specification of $\mathrm{Z}+\mathrm{F}$ 3D laser scanner

\begin{tabular}{lc|}
\hline maximum distance & $79 \mathrm{~m}$ \\
minimum distance & $1.0 \mathrm{~m}$ \\
Resolution & $0.1 \mathrm{~mm}$ \\
data acquisition & $<500,000 \mathrm{pxl} / \mathrm{s}$ \\
speed & $<1 \mathrm{~mm}$ \\
Accuracy & 310 -degree arc \\
$\begin{array}{l}\text { vertical } \\
\text { revolution } \\
\text { horizontal } \\
\text { revolution }\end{array}$ & 360 -degree arc \\
\hline
\end{tabular}

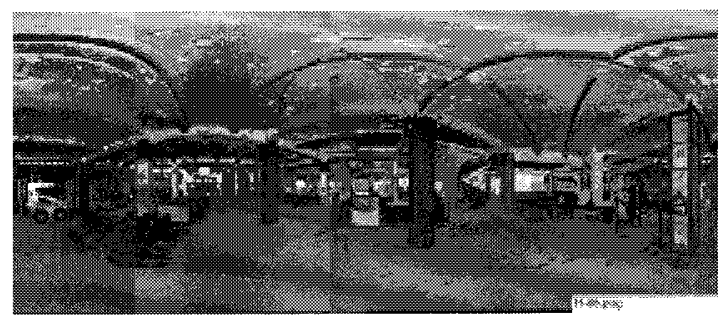

(a) $1^{\text {st }}$ Floor

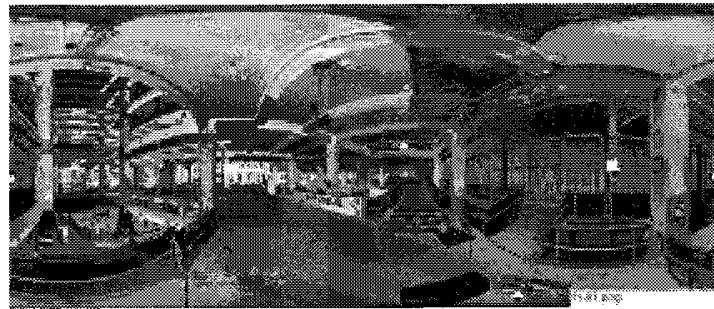

(b) $2^{\text {nd }} \quad$ Floor

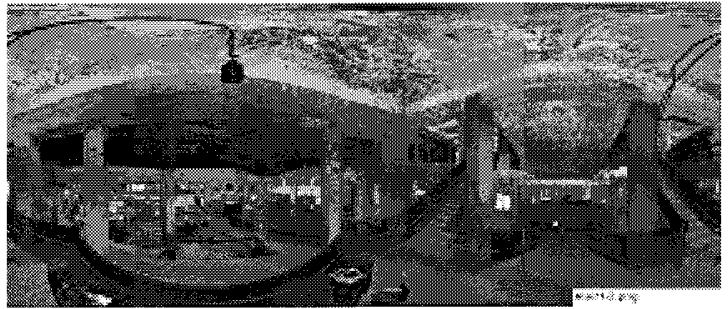

(c) Stair

Fig.2. Inside image of a remodeling department building

A laser scanner measures and records relative coordinates and transfers data from receiving apparatus to a laptop PC. The size of inside of department building is about $49 \times 59 \mathrm{~m}$ in plane size of each floor. Fig.2 shows scan image data of first and second floors and stair, which combines both floors in department building inside.

Total 96 control points were stuck on side walls and stair walkway walls of first and second floors of remodeling object and surveyed by a total station, Sokkia 2BII. Scan data were acquired respectively at several stations in first and second floor. Table 2 shows 3D coordintes of 96 control points.

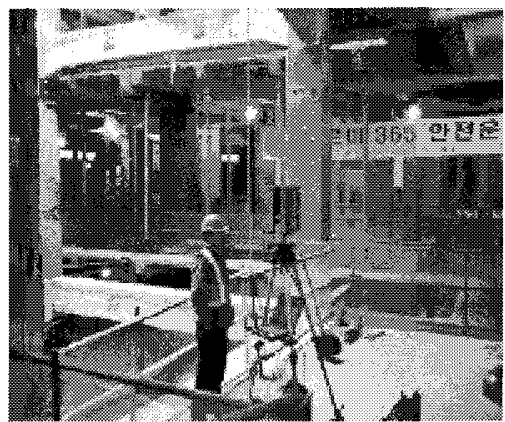

Fig.3. Data acquisition scene 
Table 2. 3D coordinates of 96 control points surveyed by a total station.

\begin{tabular}{|c|c|c|c|}
\hline Point No. & $\mathrm{X}$ & $\mathrm{Y}$ & $Z$ \\
\hline 103 & 159.797 & 111.408 & 38.304 \\
\hline 102 & 145.551 & 129.992 & 37.176 \\
\hline 104 & 163.695 & 129.754 & 37.934 \\
\hline$\cdots$ & $\ldots$ & $\cdots$ & $\cdots$ \\
\hline 150 & 164.486 & 148.907 & 45.445 \\
\hline 151 & 181.787 & 152.477 & 46.459 \\
\hline 152 & 190.292 & 140.038 & 46.540 \\
\hline$\cdots$ & $\ldots$ & $\ldots$ & $\cdots$ \\
\hline 209 & 152.483 & 129.879 & 33.998 \\
\hline 210 & 160.156 & 156.341 & 32.791 \\
\hline 211 & 158.109 & 148.214 & 34.234 \\
\hline
\end{tabular}

\section{REGISTRATION AND GEOREFERENCING}

Continuous scan clouds of a object should pass through the registration procedure which is related to relative spatial matching and joining and the georeferencing procedure which is related to transformation to absolute coordinates. Registration is a procedure which compute relative positions by aligning scan data measured at different stations as shown in Fig. ${ }^{5)}$. This is the process that performs coordinate transformation to form the same coordinate system. Here coordinate transformation is determined by translation and rotation factors in space.
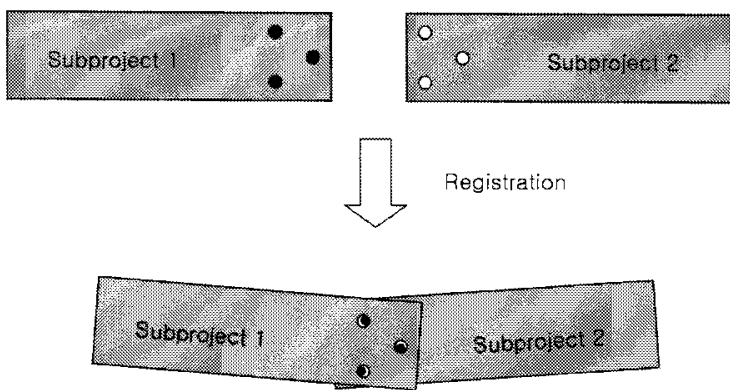

Fig. 4. Registration of continuous scan data

Scan data which are aligned in the same coordinate system after registration are transformed into absolute coordinates by combining with ground control points through the georeferencing as shown in Fig. $5^{5}$.

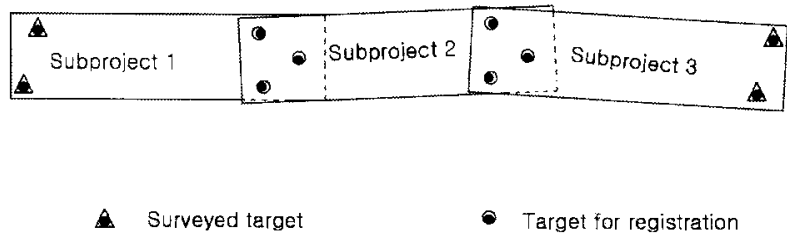

Fig. 5. The principle of georeferencing
Rapidform was used as a 3D scan modeling software for post-processing laser scan data for reverse engineering or inspection. It makes 3D scanning an extremely powerful tool for a variety of applications and is used in manufacturing, R\&D, quality inspection, medical research, civil engineering and more

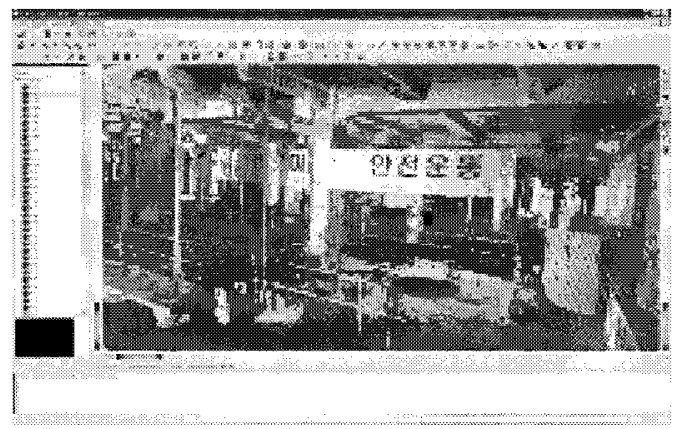

Fig. 6. Scan data acquired at a station of $1^{\text {st }}$ floor

\section{DATA PROCESSING}

Point-clouds with 3D coordinates(X,Y,Z), which are generated from the data of the object surface, are edited for deleting unnecessary data by $3 \mathrm{D}$ modeling sofrware, Rapidform ${ }^{9}$. And then through registration and merging processing on Rapidform software, scan data acquired respectively at several stations in first and second floor were merged in a unified coordinate system using 96 control points which were arranged properly. Fig.7 shows the merged scan data of 1st floor and Fig.8 shows merged scan data of 1st and 2 nd floors on the Rapidform S/W.

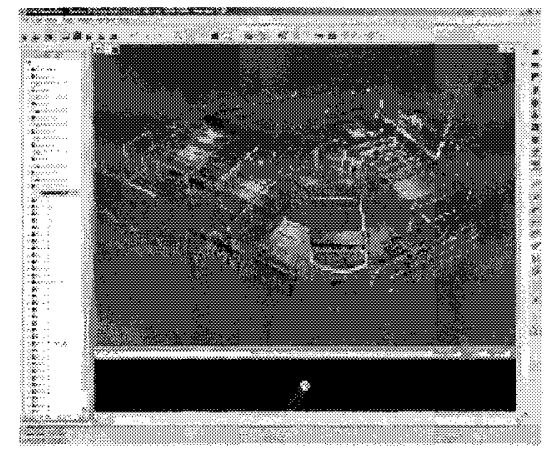

Fig.7. Merging scan data of $1^{\text {st }}$ floor

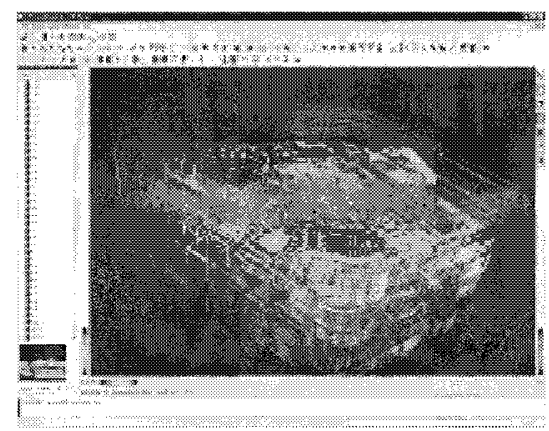

Fig.8. Merging scan data of $1^{\text {st }}$ and $2^{\text {nd }}$ floor 
Fig.9 shows the slicing function of the Rapidform for extracting a plane drawing from 3D model data. The black plane shows the position of a cross section in this figure.

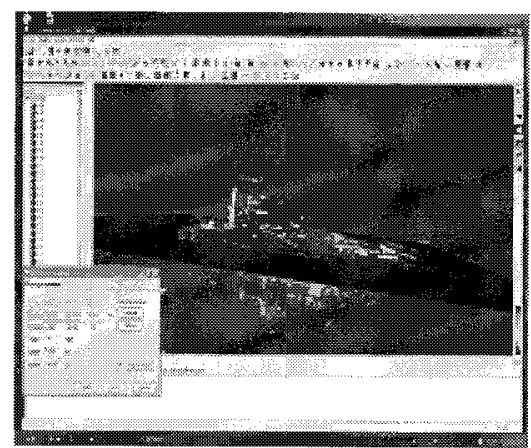

Fig. 9. Slicing for extracting plane drawing using postprocessing software

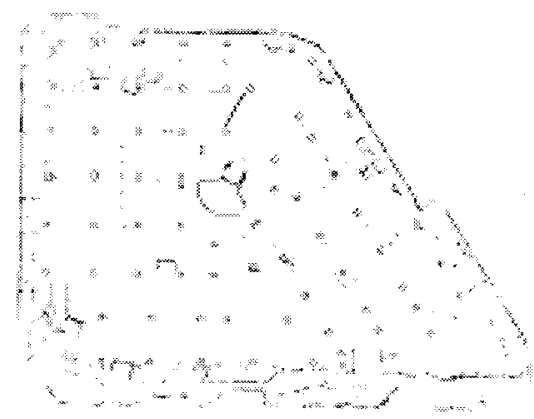

Fig.10. Extraction of a plane figure from combined scan data

The plane figures were extracted as you see in Fig. 10 by slicing of a combined scan data and Fig.11 shows a plane design drawing at that time of construction.

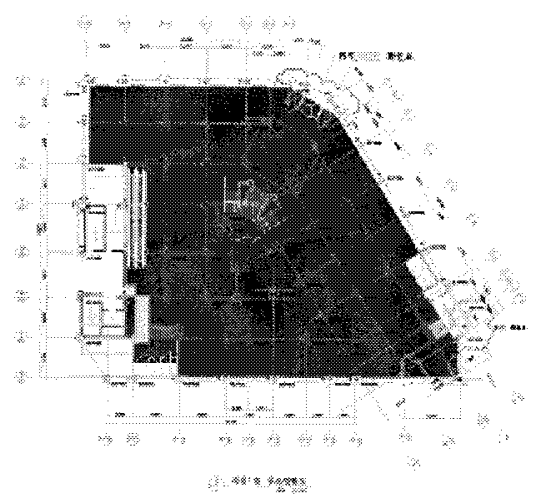

Fig.11. Design drawing at that time of construction

For recording or preserving the shape information of a building, the data of not image concept but digital concept are required and so 3D shape data should be produced applying reverse design technology. Laser or optical noncontact measurement methods are so speedy and precise that it can be utilized as a optimal method for 3D measurement of a intricate building interior.

\section{CONCLUSION \& DISCUSSION}

The products obtained by laser scanning are point-cloud data which are composed of a great number of points. Because the products themselves are nothing but basic data for the end products, they are tranformed into 3D data which they require only when they go through postprocessing by proper softwares

Remodeling execution of a building interior is in need of accurate drawing maps. However if design drawing at that time of the building constretion are lost or damaged or it is different from reality in dimension, resurvey of interior is needed. In this case laser scanning and drawing data extraction function of related processing software raise greatly work efficiency.

\section{REFERENCES}

[1] S.H. Lee, Y.C. Hwang, S.H. Kim, S.R. Sim, and T.Y. Jeong, "A Study on Precision Measurement of Rock Joint Using 3D Laser Scanner", Explosives \& Blasting, Korean Society of Explosives \& Blasting Engineering, Vol.22, No.3, 2004, pp. 103-111.

[2] I.S. Lee, D.K. Tcha, and S.J. Kim, "Cadastral Reconnaissance Surveying in Land Consolidation Area Using Terrestrial Laser Scanner", The Journal of KSCE(D), Korean Society of Civil Engineers, Vol.27, No.2, 2007, pp. 233-239.

[3] H.S. Han, H.G. Sohn, and J.S. Kim, and S.K. Woo, "Examination of Construction Site Using 3D Laser Scanner", Joint Conference of Korean GIS Societies, June, 2008, pp. 525-528.

[4] J.D. Lee, C.H. Do, and S.H. Han, "3 Dimensional Modelling of a Old Architecture Using a Terrestrial Laser Scanner", 2007 Fall Conference of Korea Contents Association, The Korea Contents AssociationVol.5, No.2(1), 2007, pp. 30-34

[5] J.C. Lee, Y.H. Lee, D.Y. Moon and D.J..Seo, "Extraction of Coastal Topography Using Terrestrial Laser Scanning Technique", Proceeding of Korean Society of Surveying, Geodesy, Photogrammetry, and Cartograph, Jeju, Korea, 2007, pp. 435-438.

[6] A. Brzank, P. Lohmann, C. Hejpke "Automated Extraction of Pair Wise Structure Lines Using Airborne Laser Scanner Data in Coastal Areas,", Congress of ISPRS, WG III/3, III/4, Netherlands, September, 2005.

[7] Fröhlich, C.; Mettenleiter, M., "Terrestrial Laser Scanning - New Perspectives in 3D Surveying", International Archives of Photogrammetry, Remote Sensing, Spatial Information Science, Vol.XXXVI-8/W2.

[8] http:/www.slope.co.kr/image/scanner3dsd.pdf.

[9] http://www.rapidform.com/index.htm. 


\section{Jin-Duk Lee}

He received the B.Eng. in agricultural engineering from Chungnam National University, Korea in 1983 and also received M.Eng.., Ph.D. in civil engineering from Chungnam National University, Korea in 1986 and 1992 respectively. Since 1990 , he has been with Department of Civil Engineering, Kumoh National Institute of Technology. His main research interests include geographic information system, digital photogrammetry, remote sensing and digital mapping.

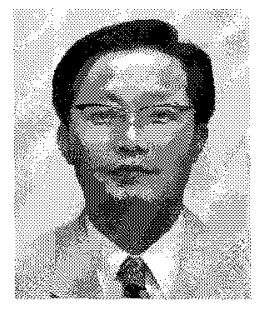

\section{Seung-Hee Han}

He received the B.Eng., M.Eng.. in civil engineering from Chungnam National University, Korea in 1984 and 1987 respectively. He also received $\mathrm{Ph} . \mathrm{D}$. in civil engineering from Chungnam National University, Korea in 1993. Since 1993, he had been with Department of Civil Engineering, Cheonan National Industrial College and since 2005, he has been with Department of Civil Engineering, Kongju National University. His main research interests include geographic information system, digital photogrammetry, remote sensing.

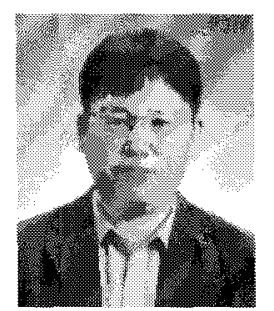

\section{Jae-Bin Lee}

$\mathrm{He}$ received the B.Eng. in civil engineering from Kumoh National Institute of Technology(KIT), Korea in 1993,. He performs for the degree of Master in civil engineering of KIT.

$\mathrm{He}$ is a CEO of Dongsan Survey ENC Co His main research interests include programming and system development for hydrographic surveying. System, Terrestrial Surveying and so forth. 